\title{
RF IMPEDANCE MEASUREMENTS ON THE DARHT-II ACCELERATOR INTERCELL ASSEMBLY*
}

\author{
W.M. Fawley" and S. Eylon, LBNL, Berkeley, CA 94720-8211 USA \\ R. Briggs, SAIC, Walnut Creek, CA 94596 USA
}

\begin{abstract}
We report upon recent experimental measurements made of RF properties of the intercell assembly of the second axis accelerator[1] of Dual Axis Radiographic Hydrodynamic Test (DARHT) facility at LANL The intercells provide both pumping and diagnostic access to the main DARHT-II beamline. Their design includes a pumping plenum separated from the main beam pipe by return current rods together with RF shielding provided by a copper-coated stainless steel mesh. Measurements using the twin lead technique (see Ref. [2]) at low frequencies $(f \leq 200 \mathrm{MHz})$ suggest a constant value for the ratio $\eta$ of the radial and azimuthal magnetic field components to which the transverse impedance is linearly related. We find that these results compare favorably to predictions from a simple analytic, lumped circuit model which includes the effects of the mesh and return current rods. We also present RF loop-to-loop frequency scans above beam pipe cutoff $(\sim 600 \mathrm{MHz})$ showing the existence of many RF modes with relatively high $Q^{\prime}$ s.
\end{abstract}

\section{INTRODUCTION}

The main function of the intercell assemblies on the DARHT-II accelerator is to provide both adequate pumping and diagnostic access to the beamline between cell blocks. The intercell design also must provide a low impedance path for the electron beam return current, and adequate RF shielding of the beam from possible high $Q$ $\mathrm{RF}$ modes in the pumping plenum box. In this paper we describe recent measurements of both the impedance and the RF mode structure of the intercell assembly. We first describe the intercell design and then summarize analytic predictions for the effective impedance of the wire mesh. We then describe our measurement setup and technique followed by the twin lead impedance results at low and high frequencies.

\section{EVOLUTION OF THE DARHT-II INTERCELL DESIGN}

Originally, the intercells in DARHT-II were to be spaced every eight accelerator cells but then, due to ion hose growth concerns, the spacing was reduced to every six, thus increasing the pumping effectiveness by a ratio $(8 / 6)^{2}$. The electron beam current return path across the intercell is provided by eight, 1/4"-diameter, stainless steel return current rods spaced uniformly in azimuthal angle around the pumping plenum opening at the beam tube radius $(=5 ")$. Then non-specific concerns about the

\footnotetext{
*Work supported by U.S. DOE under Contract No. DE-AC03-76SF00098. "1WMFawley@lbl.gov
}

possible existence of high frequency, high $Q$, RF modes in the pumping plenum led to a request that RF shielding consisting of a conducting mesh be placed around the return current bars. The selection of wire mesh parameters involved tradeoffs, since the "geometrical transparency" of this mesh needed to be as high as possible for pumping effectiveness but the larger the mesh holes, the larger the effective RF impedance. Some initial analysis and calculations were then done concerning the impedance of a mesh. The mesh chosen was a square 20-mm grid composed of $0.56-\mathrm{mm}$ diameter stainless steel wire resulting in a geometrical transparency of about $94 \%$. It was then speculated that since the (effective) dipole $L / R$ time of the SS mesh might be less than one microsecond, transverse resistive wall type amplification of low frequency corkscrew displacements, etc., might be an issue and the mesh was coated with approximately $1 \mathrm{mil}$ of copper to reduce its resistance. In all, there are 11 intercells in DARHT-II, 4 with and 7 without solenoid magnets. Figure 1 shows a CAD representation of the intercell structure and its components.

\section{ANALYTIC RF IMPEDANCE MODEL FOR THE WIRE MESH}

We developed a simple analytic model to estimate the effective RF impedance of the wire mesh/reutrn current rod assembly. First, we presume that at RF wavelengths much greater than the wire spacing $s$, the mesh impedance is dominated by the self-inductance (which is associated with the time-dependent magnetic field in the mesh openings) and the wire resistance. The transverse interaction impedance of a gap region of length $w$

$$
Z_{\perp}=-j \sqrt{\frac{\mu}{\varepsilon_{n}}} \frac{w}{\pi b^{2}} \eta
$$

where $b$ is the beam pipe radius and $\eta$ is a complex dimensionless number giving the ratio of radial magnetic field in the gap to azimuthal magnetic field at the wall several beam pipe radii away from the gap. For a meshcovered gap at relatively low frequencies, $\eta$ scales as:

$$
\eta \equiv-{ }_{r}^{H_{r}{ }_{r}=b}=-\begin{array}{r}
s \Lambda_{m} \\
\eta_{-} h
\end{array}
$$

Here $K_{\mathrm{z} 0}$ is the surface-area-averaged dipole return current, $s$ is the mesh spacing, and $\Lambda_{m}$ is a logarithmic factor relating the magnetic energy around the mesh wires (with radius $\rho$ ) to their lumped inductance:

$$
\Lambda^{m} \approx \ln \left(s_{\curvearrowright}\right)
$$


Note that this formulation is for TM-like modes; since $E_{\theta}$ is shorted out by the wire mesh, TE-like modes should be strongly suppressed. The 8 thick, return current rods also contribute to the inductance; putting this in parallel with the mesh inductance reduces $\eta$ by $\sim 1.5$ resulting in an estimated final value of $|\eta|=0.045$. A key result here is that the low frequency impedance is predicted to be independent of frequency in the range of validity of the model.

\section{EXPERIMENTAL SETUP AND TECHNIQUE}

A "twin-lead/twin loop" technique was used to measure the transverse RF impedances of the intercell structure, similar to that previously used to measure DARHT-II accelerator cell transverse impedances (see Refs. 2 and 3). A production intercell was mounted on a support stand with 3-foot long, 10"-inner diameter "surrogate" beam tube sections clamped on both ends. A 2-meter long twin lead made of two, 1/2"-diameter copper tubes with their centers transversely spaced $1.5^{\prime \prime}$ apart was placed on the longitudinal axis of the system The twin lead was excited by the $+/$ - output from a balun, itself driven by output from a HP-8751A network analyzer. The twin lead was terminated at the other end in its characteristic (TEM mode) impedance (about 214 ohms). A "spider" of resistors connected the midpoint of the 214-ohm resistor to the tube (ground) in order to match any "monopole" (i.e. common mode) currents $(+,+)$ that might be generated (see Ref. [3]). Termination in the characteristic impedance ensures that the dipole RF fields will be in the form of a traveling wave, which simulates the transverse fields of a relativistic beam with a transverse displacement oscillating (in the laboratory frame) at frequency $f$.

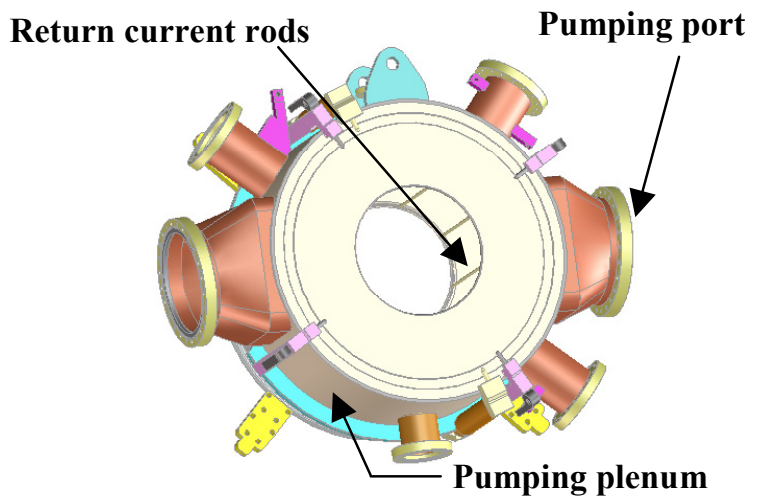

Fig. 1 - Depiction of DARHT-II Intercell Assembly

A small loop (a 3/8" ID loop made with a miniature coax wrapped in a circle) was used for the impedance measurements over the full frequency range (i.e. 1-2000 $\mathrm{MHz}$ ) highlighted in the following section. Each loop was attached in one of two possible configurations to the end of a solid copper coax and then inserted into the pumping plenum through the one of the diagnostic ports (see Fig. 1). One configuration had the loop axis perpendicular to the coax line and was used to detect the $B_{\theta}$ or $B_{\mathrm{Z}}$ field components. The other configuration involved the loop being bent at 90 degrees in order to detect $B_{r}$. Each type of loop could be inserted through the wire mesh to measure fields on the inside "beam tube" region as well as the outside pumping plenum region. The twin lead was oriented azimuthally in the tube so that one of the diagnostic ports would be aligned with the maximum $B_{\mathrm{r}}$ component (perpendicular to the plane of the twin lead), while another (i.e. orthogonal) diagnostic port would be

located at the maximum of $B_{\theta}$. In order to measure the low level RF signals in the plenum region, we had to wrap tin foil shields over the twin lead input and termination ends to minimize stray RF emission.

To make a quick survey of the resonant frequencies and $Q$ 's of RF modes localized primarily in the plenum pumping box region, we used the usual loop-to-loop coupling method (see, e.g., Ref. [3]). The $B_{r}$ and $B_{\theta}$ loops were each inserted radially about halfway into the plenum through different diagnostic ports, with the feed coax clamped to the side of the port (the twinlead assembly was removed for these particular measurements). The $B_{\theta}$ loop was driven by the network analyzer while the $B_{r}$ loop was used as the pickup.

\section{RESULTS}

Figures $2 \& 3$ show typical frequency scan data obtained with the $B_{\theta}$ and $B_{r}$ loops. The RF field amplitude scale changed by a factor of 20-40 between the measurements made inside the mesh compared with those made outside.

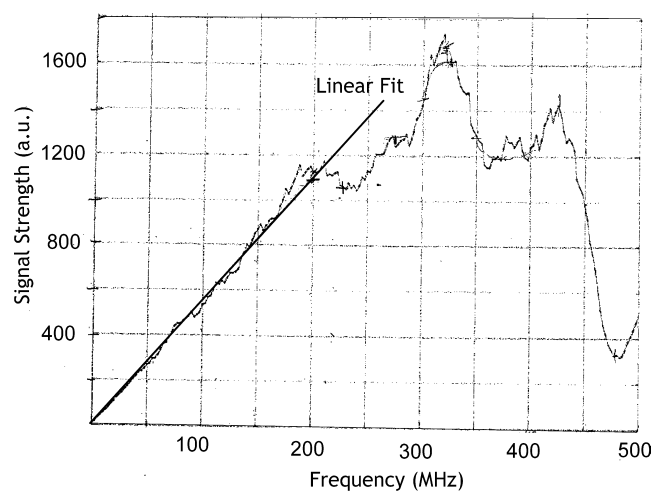

Fig. $2-B_{\theta}$ measured inside wire mesh at $r=4.625 "$.

Both inside and outside the mesh,, the loop signals rose linearly with frequency up to about $200 \mathrm{MHz}$ at all radial positions. This behavior implies that $\eta$ is independent of frequency over this "lower frequency band", in agreement with the predictions of the simple analytic model discussed above. At frequencies above $\sim 200 \mathrm{MHz}$, the RF magnetic fields of the twin lead inside the mesh showed both a general decrease in amplitude and an oscillatory structure versus frequency. These oscillations are likely 
due to a combination of mismatches at the higher frequencies and excitation (possibly due to stray inductances) of common mode currents on the twinlead.

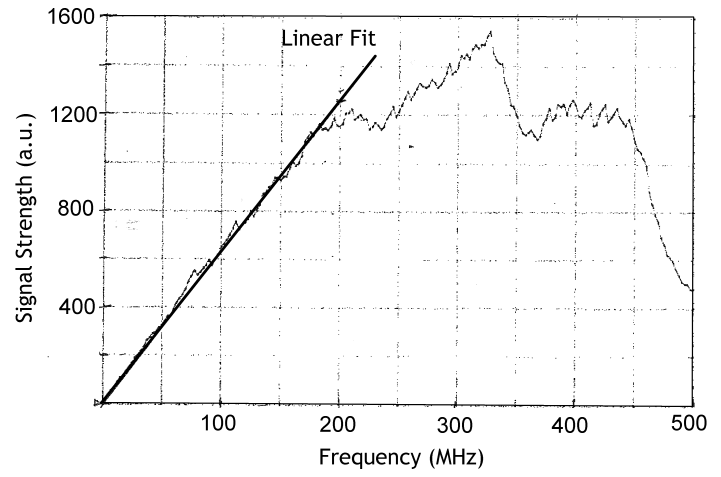

Fig. 3 - $B_{\mathrm{r}}$ measured inside wire mesh at $r=2.875^{\prime \prime}$.

The fields deep inside the plenum, on the other hand, drop little at higher frequency. This implies that the RF field penetration through the mesh and the magnitude of the transverse impedance $(\propto \eta)$ both increase with frequency in this range.

A value for $\eta$ may be determined by extrapolating a value for $B_{r}$ inward to the mesh radius from data outside the mesh and a value for $B_{\theta}$ outwards to the mesh from data inside the mesh. The result as a function of frequency is displayed in Fig. 4 and shows virtual no dependence upon $f$ below $200 \mathrm{MHz}$ as expected analytically.

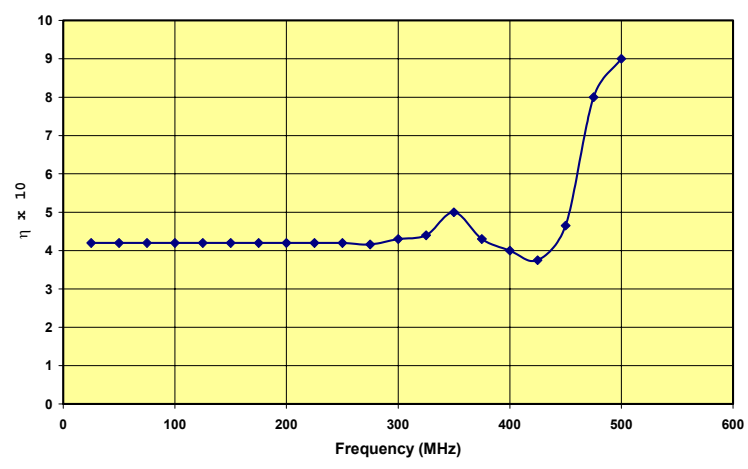

Fig. 4 - Reduced $\eta$ factor from twinlead data

\section{HIGH FREQUENCY MEASUREMENTS}

We made several twin lead high frequency (0.5 to 2.5 $\mathrm{GHz})$ scans of the network analyzer pickup signal $\left(\mathrm{S}_{12}\right)$ fin the intercell plenum. For some of the "stronger" resonances, $Q$ 's were measured and found to be as large as $\sim 800$. As might be expected from applying a simple analytic pillbox model with the 6.5 " radial separation of the mesh from the outer plenum boundary at 11.5", the resonant frequencies of the modes appearing in these scans are all above $800 \mathrm{MHz}$. Figure 5 shows a sample measurement; in this particular case, conducting plates were clamped over the pumping ports. When the conducting plates were removed, the $Q$ 's dropped $\sim$-fold. The $1.69 \mathrm{GHz}$ mode was common to both cases with a $Q$ value of $(680,140)$ for plates (on,off). Since the actual pump boundary is relatively far away, we believe the "plate-off" case is more likely to be representative of the actual situation in a working DARHT-II intercell. Due to time constraints, we were unable to investigate the mode field structure and could not determine the relevant $Z_{\perp}$.

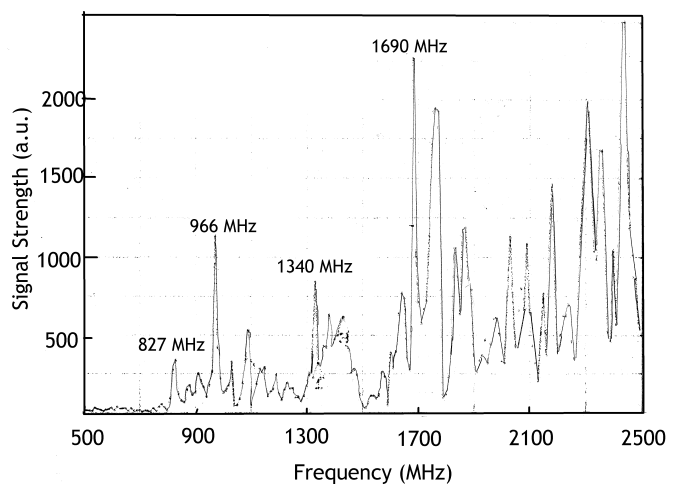

Fig. 5 - High frequency scans of the intercell plenum

\section{CONCLUSIONS}

Our twin lead measurements at frequencies below beam pipe cutoff $(690 \mathrm{MHz})$ show no indication of dangerous resonant modes. In general the impedances are low with $\eta$ values ranging from 0.04 to 0.08 . Compared with an individual accelerator cell, each intercell has a reactive impedance $\sim 3$ times smaller. At the lower frequencies the measurements are in good agreement with simple analytic models of the shielding effects of the wire mesh.

From our preliminary RF mode studies at frequencies above cutoff we found that there is a "forest" of relatively high $Q$ modes in the intercell above $800 \mathrm{MHz}$ or so. It is possible (but not at all certain!) that one of these could couple to the beam (although early commissioning results at $\sim 1.3 \mathrm{kA}$ do not show any obvious beam transport difficulties from such high frequency modes). We suggest that the DARHT-II commissioning team insert some RF loops in diagnostic ports of one or more of the intercells to monitor the RF behavior.

\section{REFERENCES}

[1] C. Ekdahl et al., "DARHT-II Commissioning Results", Paper ROAC008, this conference.

[2] R. Briggs et al., "Transverse Impedance Measurements of the DARHT-2 Accelerator Cell", PAC2001, Chicago, June 2001, p.1850.

[3] D. Birx, R. Briggs, and L. Reginato, "BBU Impedance Measurements on the DARHT-2 Cell", LBNL-42876, 1999. 